\title{
Theoretical Modelling of Charge Transport Properties of Individual Single-Wall Carbon Nanotubes
}

\author{
${ }^{1 *}$ G. Ijeomah, ${ }^{2}$ F. Samsuri, ${ }^{3} F$. Obite and ${ }^{4}$ M.A. Zawawi \\ ${ }^{1,2}$ Faculty of Electrical \& Electronics Engineering, Universiti Malaysia Pahang, 26600, Pekan, Pahang, \\ Malaysia \\ ${ }^{3}$ Department of Physics, Faculty of Physical Sciences, Ahmadu Bello University, Zaria, Nigeria \\ School of Electrical \& Electronic Engineering, Universiti Sains Malaysia, 14300 Nibong, Tebal, Penang, \\ Malaysia \\ *Email: guijeomah2@gmail.com
}

\begin{abstract}
Experimental projection of transport properties of semiconductor devices faces a challenge nowadays. As devices scale to nanometre scale range, the classical transport equations used in current device simulators can no longer be applied. Conversely, the use of a more accurate and better nonequilibrium green function (NEGF) is limited by the fact that it requires excessive quantum of memory and computational time, having quasi-separable matrices that are extremely convoluted to solve. This work exploits the Boltzmann Transport Equation (BTE) to assess the transport properties of carbon nanotubes. Previous works on solving the BTE have employed either a stochastic method or an approximate method, both of which do not possess the necessary properties for practical device applications. Therefore, this work represents the first direct theoretical solution of the BTE for onedimensional carbon nanotubes that can be utilized for practical device applications. The complete spectrum of transport in CNTs extending from ohmic to high-field through ballistic transmission is examined to delineate plethora of transport properties. The transport for arbitrary values of the electric field is based on the BTE applied to experimental data on CNTs. In the limit of low field, the mobility expressions are obtained in terms of the mean free path $(\mathrm{mfp})$ that is distinctly shorter than the length of the sample. The ohmic resistance is quantized a value of $6.453 \mathrm{k}$-ohms consistent with experimental findings with transmission approaching unity as channel length shrinks below the carrier mfp. The emission of a quantum was observed to lower the saturation velocity that is independent of scattering and hence ballistic. Transition to ballistic domain was found to occur when the channel length is scaled below the ballistic limit that is shown to be the extended version of the long-channel mfp modulated by injections from the contacts, yet the mobility degrades. The mobility degradation is shown to be the cause of resistance quantum in the low-channel length limit. These findings are important in predicting the transport properties of low-dimensional CNTs.
\end{abstract}

Indexed Terms- Electron transport, carbon nanotubes, Boltzmann transport equation, quantum conductance.

\section{INTRODUCTION}

The last few decades have witnessed a phenomenal growth in nanotechnology research. One of the most exciting disciplines to emerge from this effort is nanoelectronics, where a plethora of possibilities are emerging in the form of sensors, actuators and field effect transistors (FETs), each characterized by feature size of the order of a few nanometres. This is propelled by the discovery of new materials and 
creation of smart synthesis approaches that enable robust design and fabrication at such a tiny scale. Carbon nanotubes (CNTs) are at the core of these novel materials, revealing outstanding properties at the nanoscale.

There are two categories of carbon nanotubes (CNTs), namely single-wall carbon nanotubes (SWCNTs) and multi-wall carbon nanotubes (MWCNTs). SWCNTs can further be categorized into two, namely chiral and achiral carbon nanotubes. Carbon nanotubes whose mirror images are identical to their original structures are said to be achiral, and chiral otherwise. Achiral CNTs can further be categorized into two species, namely armchair and zigzag carbon nanotubes. Individual single-wall carbon nanotubes are the focus of interest in this research, and investigating their carrier transport properties is the main goal of this work.

Our aim to reduce the dimension of electronic devices and improve device performance has led us into the low-dimensional regime, where quantum mechanical effects predominate and thus require comprehensive investigation. The low-dimensionality of carbon based devices such as carbon nanotubes and quantum dots results in intriguing electronic transport properties that make them attractive candidates for future technological applications. The starting point of synthesizing nanoscale devices is to establish a fundamental understanding of their electronic transport properties. Essentially, a realistic and precise prediction of the electronic transport properties of these devices in low-field and high-field regimes is required to model efficient and high performance real devices.

A long standing issue in carbon nanotube devices is the nature of the electron transport traversing these devices when they are scaled below the scattering-limited threshold. The prediction of the transport properties of carbon nanotubes, that is, the response of roving carriers to the application of electric field, is perhaps a promising method to characterize materials properties at the nanoscale. In general, the nature of transport in nanoscale devices is a function of the active region of the device. An important characteristics of nanoscale contacts is the presence of schottky barriers [1] at the electrodenanoscale interface which significantly limits device conductance and dwarfs current delivery capability - a key factor in device application and performance.

Various groups have reported the transport properties of carbon nanotubes [2-4], and investigated conductance variations in CNTs when used in field effect transistor applications [5]. Furthermore, the charge transport properties of these materials have been investigated from both analytical and experimental perspectives [6]. However, the charge transport models of individual single-wall carbon nanotubes have not been fully represented. Moreso, the existing experimental techniques to study the charge transport in these devices do not come without enormous challenges owing to the presence of defect in the CNT lattice structure [7], prompting the need for simulation interventions.

Quantum effects play out in low-dimensional regimes, and modulates the operational performance of the various transport regimes, including low-field to high-field, transmission to drift diffusion, to ballistic regimes. This has particularly fuelled the relentless hunt for completely novel devices that function solely on the basis of these quantum effects, and the one-dimensional single-wall carbon nanotubes fit very well in this aspect.

Transport properties of individual carbon nanotubes is interesting because of their intriguing and unique electronic geometry. There have some research works on the experimental prediction of transport properties of carbon nanotube ropes [8] and bundles [9]. Transport measurements became possible for single-wall carbon nanotubes (SWCNTs) and multi-wall carbon nanotubes (MWCNTs). SWCNTs usually manifests large coulomb effects apparently due to the presence of nonideal contacts

In this new research, we develop a theoretical simulation model to investigate the charge transport properties of individual single-wall carbon nanotubes. The developed model exploits the wellestablished Boltzmann Transport Equation (BTE) method but with an added feature to bypass the approximation phase - a major limitation of previous works on BTE. Section II discusses the literature of carrier transport in carbon nanotubes, including the concept of scattering as carriers traverse the length scales of the nanoscale devices. Section III presents the method and specific steps employed in linearizing and discretizing the BTE both in energy and momentum to achieve self-consistency when coupled to Poisson equation. Finally, section IV presents the results of our investigation. 


\section{LITERATURE REVIEW}

The literature is replete with research reports that focus heavily of three-dimensional (3-D) assumptions of twentieth century, not valid in one-dimensional (1-D) nanostructures. Carbon nanotubes [10], are distinctly one-dimensional in the sense that quasi-ballistic transport exists owing to the propagating electron waves across the length of the channel. The carriers are transmitted in one direction as high electric fields are encountered in contrast to anticipation of current and voltage saturation owing to increased scattering.

The dispersion relation for metallic single-wall carbon nanotubes (SWCNTs) within the neighbourhood of the Fermi energy is linear, with the energetic separation between the modes at $\pm k_{F}$ in the orders of $100 \mathrm{meV}$. It is this considerable energetic separation between the one-dimensional subbands that inhibits interband scattering to a large extent even at room temperature. The presence of subbands with positive and negative slopes at $\pm k_{F}$ and $-k_{F}$ (see figure 1) permits us to write the conductance for an ideal, ballistic carbon nanotubes as,

$G=2 \frac{2 e^{2}}{h}$

where the spin degeneracy is evaluated by a factor of 2 .

The probability of scattering by impurities for three-dimensional metallic carbon nanotubes is described classically by Rutherford scattering theory [11-12],

$\tau_{i m p}^{-1} \alpha v_{F} \cdot \frac{1}{v_{F}^{4}}=v_{F}^{-3}$

where $v_{F}$ represents the Fermi velocity. The term $\tau_{i m p}^{-1}$ is independent of temperature and is proportional to the concentration of the impurity. This is based on the assumption that the velocity of the charge carriers in the interval of $4 . \mathrm{K}_{B} T$ around the Fermi energy is equal to $v_{F}$ by a good approximation. This is, in general, a valid and universal assumption for three-dimensional metallic carbon nanotubes, where $E_{F}$ is substantial comparative to $K_{B} T$. This implies that eq. (2) is valid only if the dispersion relation is linear within the energy interval of $4 . \mathrm{K}_{B} T$, which is realistic for carbon nanotubes up to room temperature and above. The magnification of the dispersion relation of metallic SWCNTs in the vicinity of the Fermi inclination at $\pm k_{F}$ is depicted in figure 1.

The arrows show the Fermi velocities of the charge carriers. Since impurity scattering is an example of elastic scattering phenomenon, a charge carrier can only be scattered in a separate state at identical energy of the CNT energy relation. The ultimate allowed states for a roving carrier in an initial state at orientation 1 are designated as 2 to 4 in figure 1 . The changes in the electron momentum of the CNT can be attributed to the presence of impurities. Moreover, the miniscule angle scattering that is present in two-and three dimensional nanostructures are not present in SWCNTs apparently due to the onedimensionality of the nanostructures. While scattering from 1 to 3 represents a forwarding scattering mechanism, scattering from 1 to 2 or 4 is a backscattering phenomenon that grows device resistance. For SWCNTs with length $L$, the two-terminal resistance is given as,

$R_{\text {imp }}=\frac{h}{4 e^{2}} \cdot \frac{L}{\lambda_{i m p}}$

where $\lambda_{i m p}$ is the elastic mean free path (mfp) that can be loosely interpreted as the average distance between impurity centers. $R_{\text {imp }}$ will be independent of temperature in a good approximation.

For phonon scattering in SWCNTs, we need to differentiate scattering by optical and acoustic phonons. Using Ozdemir formalism [13], the energy of the linear dispersion of acoustic phonons can be expressed as, 
$E_{p}=\mathrm{h} C_{p} k_{p}$

where $C_{p} \approx 10^{4} \mathrm{~m} / \mathrm{s}$ denotes the velocity of sound in a carbon nanotube. Since $C_{p}$ is approximately two orders of magnitude smaller than the Fermi velocity $\left(: 10^{6}\right)$, acoustic phonon results in a rather smaller energy changes. The final possible state 2 for an acoustic phonon scattering from an initial state 1 is delineated in figure 1 . The two states are integrated by a line whose gradient is much microscopic compared to the one with electron dispersion relation, confirming the aforementioned differential in velocity. On the other hand, scattering from the traversed dispersion region within the limit $+k_{F}$ to $-k_{F}$ is suppressed owing to the absence of the allowed, vacant states around $k_{F}$ even at room temperature. To initialize scattering from $+k_{F}$ to $-k_{F}$, therefore, about two orders of $k_{F}$ has to be supplied by the phonon.

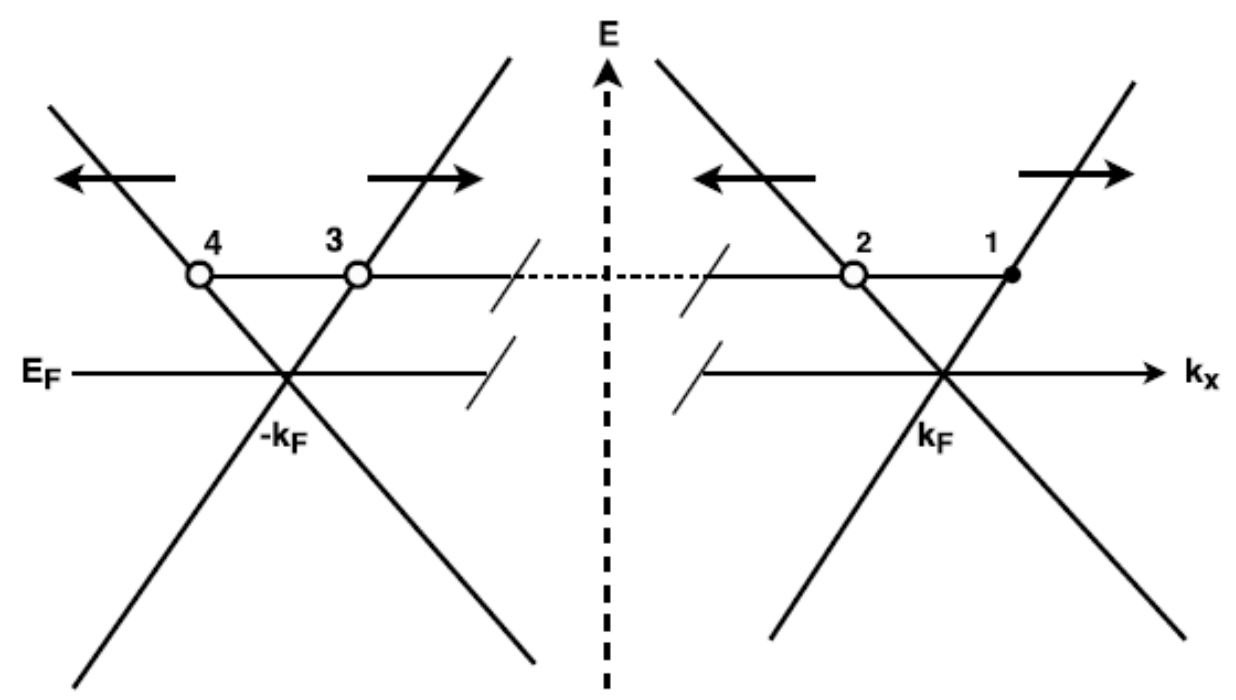

Figure 1: Schematic of elastic scattering processes on a metallic SWCNTs. Also shown is the dispersion relation within the boundary $-\mathrm{k}_{\mathrm{F}}$ to $+\mathrm{k}_{\mathrm{F}}$. Position 1 depict the inceptive state of an electron, and position 2 to 4 are the final states of the electrons after scattering events.

Since the Debye length of SWCNTs is approximately equal to $2000 K$ [14], the condition for $T<300 K$ is elucidated by the Greneisen formalism [15-16]. For three-dimensional devices, the temperature dependence of the scattering rate is given by,

$\tau_{p}^{-1} \propto T^{5}$

where the factor $\propto T$ comes from the energy switch between the carrier and phonon, one of $\propto T^{2}$ describes the miniscule angle in 3-D systems and the second $\propto T^{2}$ comes from 3-D density of phonon states. For 1-D nanosystems such as SWCNTs, the two $\propto T^{2}$ terms can be neglected because the density of states in I-D phonon manifests temperature independence, and the small angle scattering is blocked in 1-D. Accordingly, the resistance contribution due to the acoustic phonon is given by,

$R_{p}=\frac{h}{4 e^{2}} \cdot \frac{L}{\lambda_{p}}$

(6)

where $\tau_{p} \propto T^{-1}$ and $\lambda_{p}=v_{F} \cdot \tau_{p} . \lambda_{p}$ has been found to be approximately equal to $1 \mu m$ [17]. 
For small extra energies of the carriers, scattering by optical phonons can be neglected since there are no vacant states. For substantial electric fields, however, electrons in the SWCNT become "hot" after taking up energies well beyond the $K_{B} T$ limit. Interestingly, "hot"' in this sense does not imply an increase in electron velocity, instead carrier velocity remains unchanged while its energy increases due to the unusual state of the linear dispersion relation. In the case of "hot" electrons, optical phonon scattering may be the primary contribution to the overall resistance of the carbon nanotube.

Another mechanism with potential to contribute to the CNT resistance is the electron-electron scattering. This phenomenon is particularly pronounced in 1-D nanostructures. Interestingly, electronelectron scattering does not result in any appreciable change in the resistance of a standard nanostructure. This is anticipated since energy and momentum conservation can only be achieved if the magnitude of the forward scattered and backscattered carriers are in equilibrium. The dispersion relation in the vicinity of $k_{F}$ is shown in figure 2 .

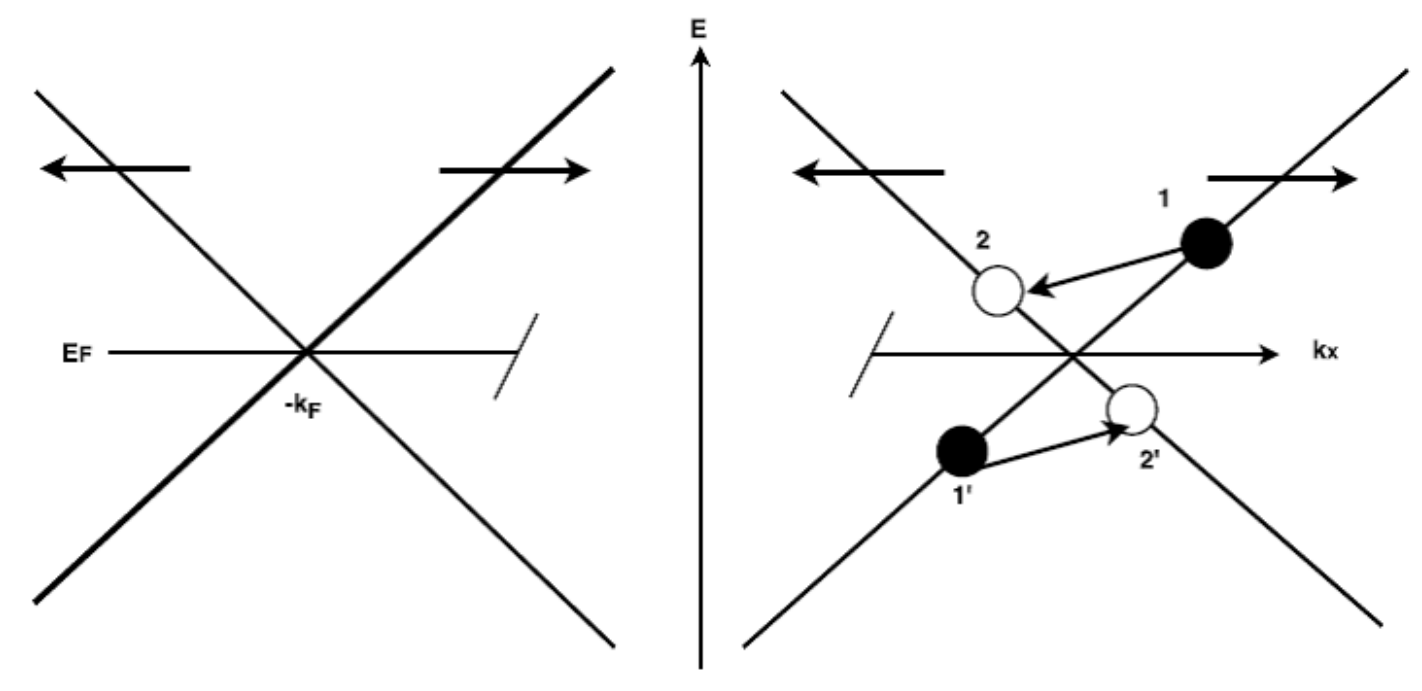

Figure 2: Electron-electron scattering in a metallic SWCNT. Also shown is the dispersion relation in the vicinity of $+\mathrm{kF}$.

Due to mode crossing, the electrons in orientation 1 and 1' which contribute to forward transport, may get scattered into orientations 2 and 2' respectively. In this process, the overall energy and momentum is conserved. The scattered carriers in orientations 2 and 2' now populate states in opposition to current, leading to an increase in resistance. As temperature rises, the quantum of allowed initial and final states increases, and so does the scattering probability. Mathematically, the linear dependence of the electronelectron scattering rate on temperature is given as [18],

$$
R_{e-e}=\frac{h}{4 e^{2}} \cdot \frac{L}{\lambda_{e-e}}, \tau_{e-e} T^{-1}
$$

where $\lambda_{e-e}$ is the mean free path between the scattering processes, and $\tau_{e-e}$ represents the inverse scattering rate. $\tau_{e-e}$ increases with increasing electric excess energy, $e V$, of the carriers due to the increase in the number of the allowed states.

As we already know, the energy bands in metallic carbon nanotubes intersect the Fermi energy [19]. Figure 3 shows the energy bands at $k_{y}=2 \pi / 3 a$ for a $(10,10)$ chiral metallic carbon nanotubes. The tube is theoretically contacted by two contact pads with quasi-Fermi levels of $\mu_{1}$ and $\mu_{2}=\mu_{1}-e V_{\text {app }}$. 
An applied electric field will cause current to flow through a single level between $\mu_{1}$ and $\mu_{2}$. A fascinating prediction for carbon nanotubes is that electron transport in these nanosystems may be ballistic [20]. In this sense, the computed conductance is a signature of a series of input and output contact pads integrated into a narrow constriction initiated by the carbon nanotube. Under this condition, the flow of current assumes a "conduction by transmission" characteristics, and the conductance becomes a characteristics of a particular system rather than the material device [21].

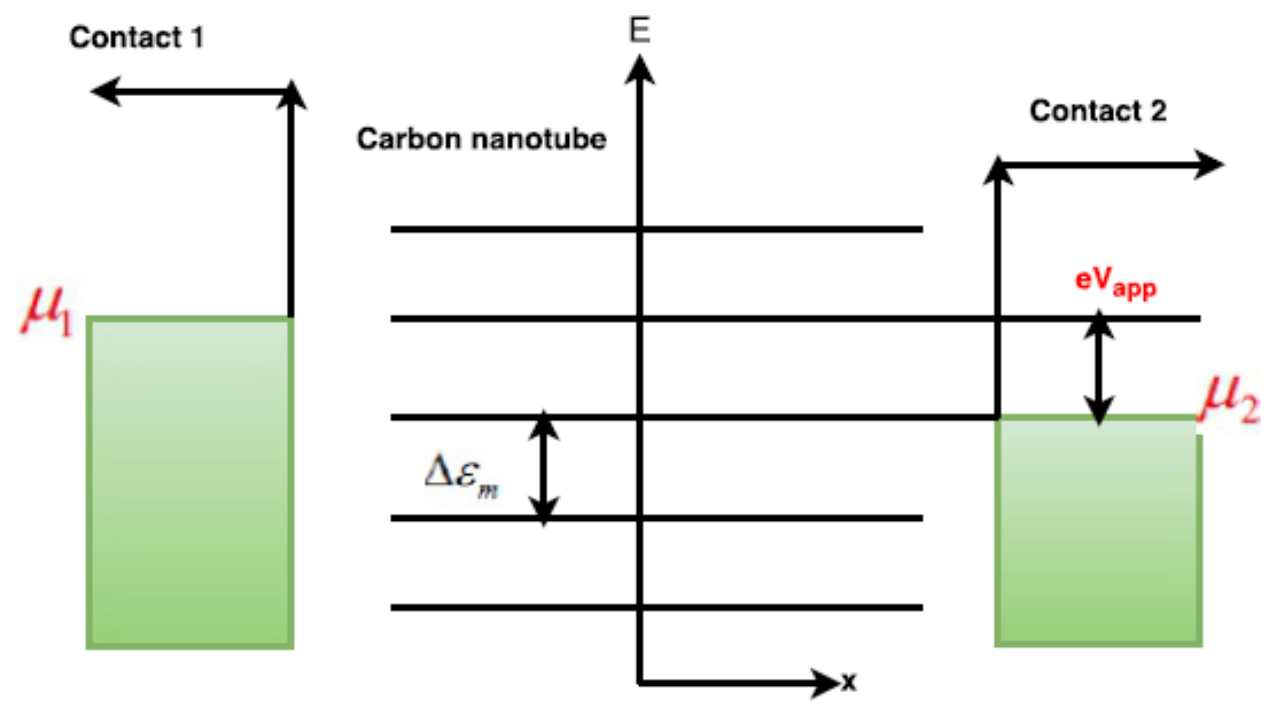

Figure 3: Schematic of transport through quantized energy level for a $(10,10)$ CNT. The applied bias of contact 2 is lowered by the energy $E_{\text {vapp }}$ with respect to contact 1 , allowing carriers to ballistically traverse the first unfilled nanotube energy levels.

This event is known as ballistic transport. Ballisticity implies that there is no scattering across the length of a carbon nanotube, and that carriers remain in local equilibrium as they traverse the contact pads. This is only possible when the length of the CNT sample is less than the scattering-limited mean free path (mfp). Further restriction requires that the diameter of the CNT $D$ be much less than the length of the sample, and comparable to the carrier wavelength at the Fermi energy $\lambda_{F}$.

The quantum resistance represents a contrasting paradigm from the usual collision-dominated resistance, where carriers are taken as itinerant particles that encounter sustained collision with the lattice. These stochastic collisions were believed to give rise to collision-dominated resistance as enshrined in Ohm's law. It follows that, in the absence of charge transport, carriers should be able to move freely across the length of the sample, and hence the resistance vanishes to zero or alternatively, the conductance becomes infinite. Is this really the case? To address this question, and gain a wealthy of knowledge regarding the primary root of conductance in a ballistic carbon nanotube, let us consider a ballistic wire connected to a reservoir of itinerant carriers as shown in figure 4 . The current $I(\mathrm{E})$ through scattering-free conductor from electron waves with energy $E$ can be calculated from the flux equation as,

$$
I(E)=\frac{\partial q(\mathrm{E})}{\partial t}=\frac{\partial q(E)}{\partial l} \frac{\partial l}{\partial t}
$$

where $\partial q / \partial l$ represents the charge density per unit length, and $\partial l / \partial t=v(\mathrm{E})$ represents the velocity of the carrier with energy $E$. If we represent the forward-moving carriers as $q^{+}$and the backward- 
moving carriers as $q^{-1}$, then the overall current from carriers with energies between $E$ and $E+d E$ is expressed as,

$$
I(E) d E=\left(\frac{\partial q^{+}(E)}{\partial l}-\frac{\partial q^{-}(E)}{\partial l}\right) v(E) d E
$$

where the plus (+) and minus (-) signs represents the forward-flowing current and backward-flowing current respectively. At equilibrium, the forward-flowing currents equals the backward-flowing current, leading to a vanishing current.

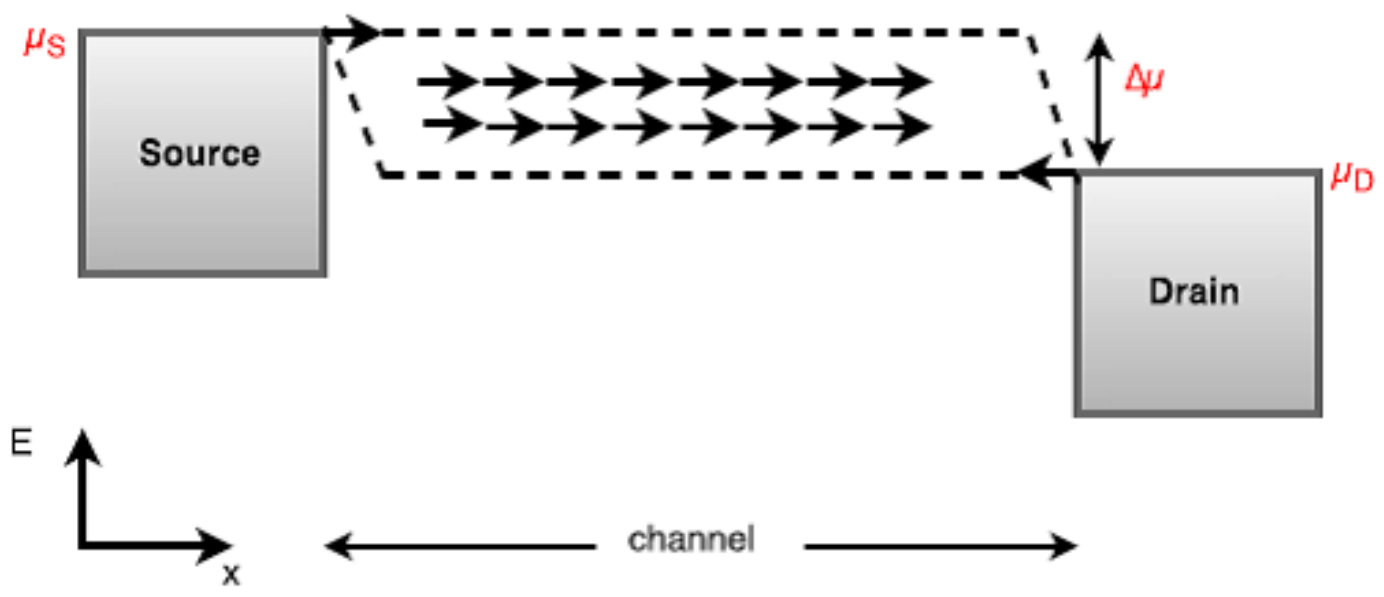

Figure 4: Band diagram of a ballistic channel coupled to source and drain reservoirs of carriers. The arrows show the direction of carrier flow from both reservoirs.

The ballistic or scattering-limited limit which reflects a length-independent conductance (or resistance) applies when the dimensions of the carbon nanotube are much less than the mfp of the carriers. This implies that, in a carbon nanotube with length $l$, much greater than the mfp, transport carriers will encounter frequent scattering with the lattice, resulting in a universal expression for conductance quantum,

$G_{q}=\frac{2 e^{2} N_{c h}}{h} T$

where $T$ is the transmission coefficient or the probability of carriers propagating through the nanotube, and satisfies the boundary condition, $0<T<1 . T=1$ is a special case of perfect transmission through the device. Scaling the nanotube from 1-D to 3-D increases the number of the propagating subbands which leads to a state of vanishing resistance. Moreover, the inclusion of the influence of carrier scattering by the lattice will definitely result in a length-dependent resistance, and subsequent recovery of ohm's law.

The perfect transmission $T=1$ is also an ambiguous issue that remains unresolved. This transmission is often confused with the tunnelling coefficient that is not applicable to micron length channels. The puzzle of a dramatic rise in resistance even for a few millivolts across the length of a scattering-free channel still remains. This surge in resistance is in direct contrast to the perception of resistance vanishing due to the elimination of scattering as the channel length of the nanotubes goes below the scattering-limited $\mathrm{mfp}$. The reason for the non-ballistic transport is usually hinged on the contact resistance. This is in spite of the fact that extraordinarily long mfps have been identified in carbon nanotubes. 
In metallic CNTs with finite temperatures and low energies with length much less than the mean free path, the exponential term of the Fermi Dirac distribution function in the second subband results in negligible contribution of quantum conductance from the second subband compared to the first subband. This implies that quantum conductance is determined only by the first subband with negligible temperature dependence. As a result, the measurement of conductance quantum is a useful technique for characterizing the nature of the contacts to the carbon nanotubes under different synthesis methods. The mobility not only degrades, but experimental results on mobility span a wide range depending on the length of the sample.

The CNT mobility $\mu$ can be deduced from the Boltzmann Transport Equation (BTE) in the stationary regime. With the application of unvarying applied bias $F$, the distribution function in the state $|k, b\rangle$ is expressed to the first order in $F$ by,

$$
\begin{aligned}
& \sum_{b^{\prime}} \int g_{b}(k)\left\{W_{k b, k^{\prime} b^{\prime}}\left[1-f^{o}\left(E_{k^{\prime}, b^{\prime}}\right)\right]+W_{k^{\prime} b^{\prime}, k b} f^{o}\left(E_{k^{\prime}, b^{\prime}}\right)\right\}- \\
& g_{b^{\prime}}\left(k^{\prime}\right)\left\{W_{k^{\prime} b^{\prime}, k b}\left[1-f^{o}\left(E_{k, b}\right)\right]+W_{k b, k^{\prime} b^{\prime}} f^{o}\left(E_{k, b}\right)\right\} d k^{\prime}= \\
& \frac{2 \pi}{L} v_{b}(k)\left(\frac{\partial f^{o}}{\partial E}\right)_{E_{k, b}}
\end{aligned}
$$

where $L$ is the length of the carbon nanotube and $v_{b}(k)=\mathrm{h}^{-1} \partial E_{k, b} / \partial k$ is the ensemble velocity along the applied electric field. The mobility is then given by,

$$
\mu=-e \frac{\sum_{b} \int g_{b}(k) v_{b}(k) d k}{\sum_{b} \int f^{o}\left(E_{k, b}\right) d k}
$$

Eqs. (11) and (12) are valid and applicable to two-dimensional devices only within the limits $d k \rightarrow d^{2} k^{\prime}$ and $2 \pi / L \rightarrow 4 \pi / S$ where $S$ is the carbon nanotube surface.

Now, let us consider some variants of carbon nanotubes, say chiral, achiral, armchair and zigzag carbon nanotubes. By default, armchair and achiral carbon nanotubes are metallic. However, zigzag and chiral nanotubes are metallic only when the chiral index ${ }^{n}$ is a multiple of 3 , and semiconducting otherwise. Since we know that carbon nanotubes can be synthesized from graphene through the roll-up process [22], their electronic bandstructures can well be related to those of graphene. In graphene kspace, the allowed 1-D k-vectors of the carbon nanotubes form a hierarchical bundle of parallel edges along the nanotube direction, the separation between the directions being inversely proportional to the diameter of the carbon nanotube. This results in the carbon nanotube bandstructure aligning in successive subbands, one for each direction. Since these conditions promote carrier transport within the energy window of a few Fermi energy around the Fermi surface, we anticipate that the carrier transport properties of carbon nanotubes can match those of graphene in three ways; by increasing a) the carrier density, b) the nanotube diameter or c) the operating temperature. In any case, the quantum of subbands in the transport energy will increase in such a way that the 1-D transport progressively coalesce into the 2-D transport so that the differentiation in the transport properties of both 1-D and 2-D nanoscale devices becomes immaterial.

An intriguing effect arises when we include the electron-electron interaction in an otherwise ballistic channel that is connected to reservoirs of self-updating carriers. In a perfect 1-D nanostructure where electron-electron interactions are viewed as Luttinger liquid [23], Maslov and Stone [24] have shown that the conductance quantum is unaffected by such interactions. Interestingly, this can be elucidated without employing complex mathematical models. This is because, as earlier established, the quantum conductance is due primarily to the dynamics of carriers emanating from the reservoir and propagating into the 1-D nanosystem. Since the carriers from the contact are self-updating, the conductance and carrier transport are due to the propagation of self-updating carriers. 
So far we have isolated the reservoir contacts to the nanosystem as being responsible for the conductance quantum. However, the role of the contacts to the nanosystem is somewhat ambiguous and is indeed not explicit in the calculation of quantum conductance. Therefore, attributing the quantum conductance to the coupling between the carbon nanotubes and the contacts is somewhat of an academic argument. Conceivably, if we carefully examine the expression for quantum conductance, it might be possible to integrate the coupling of the nanotube-contacts directly into the expression and indeed demonstrate that it is this coupling that is responsible for conductance quantum.

The ballistic transport in CNTs is limited to the low applied field regime, for which the propagating carriers can only be coupled to low-energy acoustic phonon modes [25]. By increasing the applied electric field across the CNT, the contribution of the electron-phonon initiated backscattering is progressively enhanced until saturation is reached in the current-voltage (I-V) characteristics of the device [26]. This not only triggers substantial performance limitations in the CNT current-carrying capacity, but also raises serious questions about the nature of inelastic carrier transport in these devices.

In the high-bias regime, the inelastic scattering length is predicted to be in the orders of a few tens of nanometres. Park et al. [27] predict the inelastic $\mathrm{mfp}_{l-h}$ in both low and high electric field according to,

$$
G \approx \frac{2 e^{2}}{h} \frac{1-h}{L}
$$

In the limit of low-bias, their data show that $1_{l-h}=1.6 \mu \mathrm{m}$ which can be attributed to acoustic modes, whereas $1_{l-h}=10 \mathrm{~nm}$ is assessed for applied bias of the order of $1.0 \mathrm{~V}$ which can be attributed to zone-boundary $\left(1_{z b}\right)$ or optical $\left(1_{o p t}\right)$ phonons. Other computations deduced by fitting the experimental data with semi-classical Monte Carlo models [28] led to $1_{o p t}=15 \mathrm{~nm}$ and $1_{a c}=300 \mathrm{~nm}$ for optical and acoustic modes respectively. On the other hand, within the framework of deformation potential and effective mass approximation [29], a primitive theoretical approximation of the acousticelectron phonon scattering yields $1_{a c}=24 \mu \mathrm{m}$ in the low-bias domain, whereas $1_{z b}=37 \mathrm{~nm}$ and $1_{\text {opt }}=180 \mathrm{~nm}$ were deduced in the high-bias regime. By applying Mathiessen principle, a scattering length of $l=30 \mathrm{~nm}$ was assessed. To reconcile experiment from theory, a hot carrier phenomenon was proposed [30]. While some progress has been made [31], many questions regarding the nature of electron-phonon interaction in these systems remain unanswered. Table 1 is a summary of the recent literature on carbon nanotubes in regard their transport properties.

In addition to the afore-mentioned studies, the electron-phonon interaction in CNTs has been extensively investigated from theoretical perspectives in the context of temperature-dependent conductivity in metallic CNTs [32] and temperature dependence of the band gap of semiconducting nanotubes [33]. The influence of electron-phonon interaction on the carrier properties of CNTs is thus of great importance in deepening the exceptional transport properties of CNTs.

Table 1: Summary of recent literature on carbon nanotubes

\begin{tabular}{lll}
\hline Author (s) & Research work & Reference \\
\hline Sharma and Jaggi, 2016 & $\begin{array}{l}\text { Band structure of CNT from roll-up of } \\
\text { graphene }\end{array}$ & {$[34]$} \\
Abdullah, 2016 & Chirality-induced channel breakdown & {$[35]$} \\
Evarestov et al., 2017 & Phonon dispersion in CNT & {$[36]$} \\
Xu et al., 2017; Milovanovic et al.,, & $\begin{array}{l}\text { Experimental transport in CNT, zone- } \\
\text { folding }\end{array}$ & {$[3,6]$} \\
\hline
\end{tabular}


Davoody et al., 2017

Park et al., 2017

Dasgupta and Chauhan., 2017

Zhang et al., 2016

Zhou et al., 2018
Electron transport, half-inter hall-effect

Semi-classical BTE

Ballistic transport in CNT-FETs

Quantum transport in CNTs

Computational nanoelectronics
[37]

[41]

\section{METHODOLOGY}

Semiconductor device modelling has witnessed tremendous progress in the past few years, evolving from early theoretical work into advanced kits that can simulate the electronic properties of a wide range of semiconductor devices. There is no doubt that such device simulation methods has advanced the semiconductor industry through predictive investigation and even troubleshooting of device design and fabrication [42]. As devices continue to increase in complexity and density, the underlying circuits continue to get smaller, faster and complicated. Therefore, it is anticipated that device simulation will continue to be the most realistic mechanism to design and fabricate devices with desirable features for practical applications.

The models employed to simulate these properties are basically a set of partial differential equations (PDEs) that describe the transport of itinerant carriers through the nanostructure. The solution of these set of PDEs is a function of the structure of the device and the applied electric field. Figure 5 delineates the hierarchy of these simulation models arranged in decreasing complexity. In this section, we implement a straight-forward technique to compute $f(x, v)$ for single-wall carbon nanotubes by solving the Boltzmann Transport Equation (BTE) self-consistently with Poisson solver.

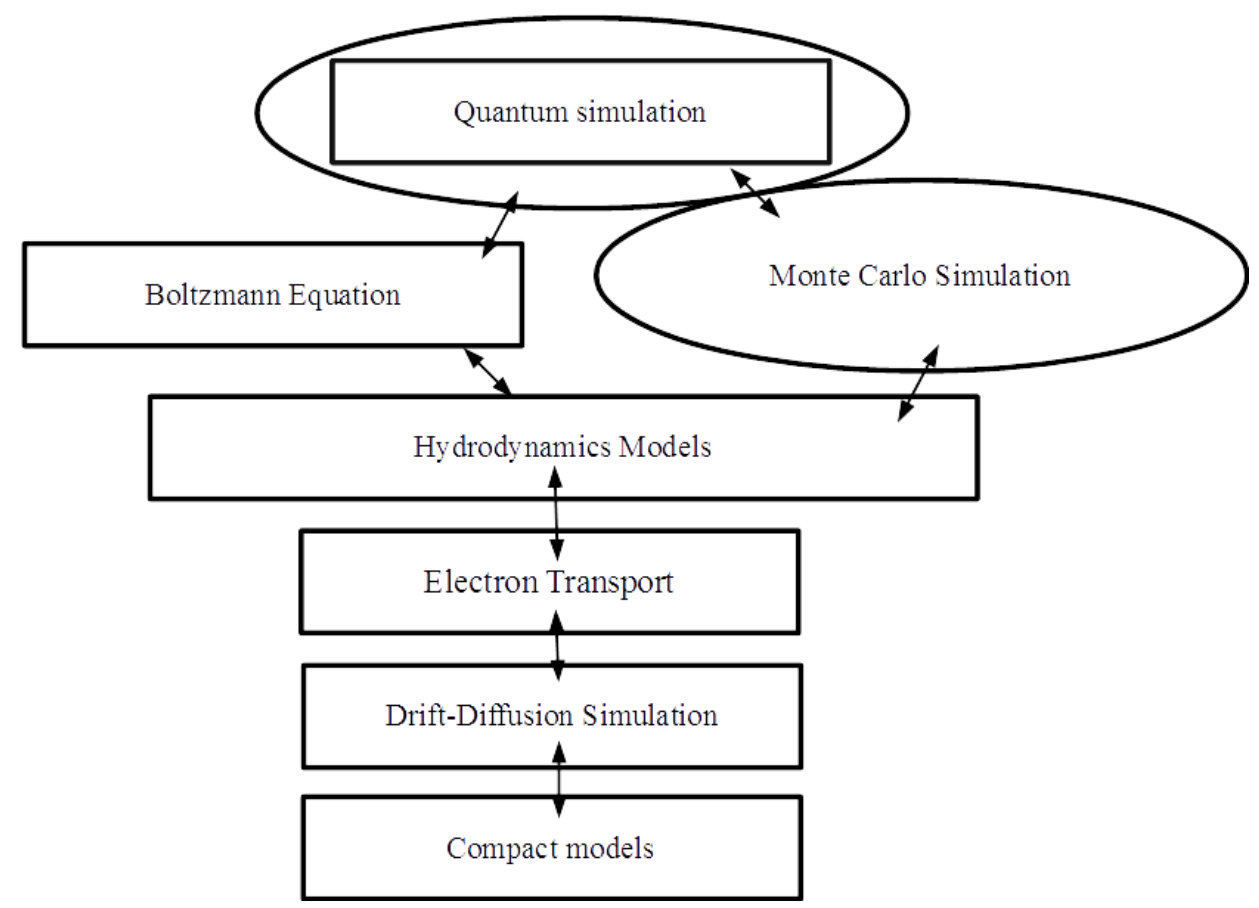

Figure 5: Flowchart of transport properties arranged in order of decreasing complexity. 
The Boltzmann Transport Equation describes the carrier transport dynamics of the distribution function, $f(r, v, t)$ under the influence of varying scattering processes, as well as magnetic and electric fields. In the absence of the magnetic field component, the 2-D phase-space, steady-state BTE for $f(x, v)$ can be written as,

$$
\frac{e E(x)}{m^{*}} \frac{\partial f}{\partial v}+v \frac{\partial f}{\partial x}=-\frac{f-f L E(x, v)}{\tau(\varepsilon)}
$$

where $f_{L E}(\mathrm{x}, \mathrm{v})$ denotes the local equilibrium distribution function appropriate for the applied electric field, density and equilibrium lattice temperature $T_{o}$, to which the function $f(x, v)$ relaxes at a relaxation rate $\tau(\varepsilon)^{-1}$. We also normalized the local density $n(x)$ using the Maxwell-Boltzmann distribution at $T_{o}$ as,

$$
f_{L E}(x, v)=n(x)\left[\frac{m^{*}}{2 \pi k T_{o}}\right]^{1 / 2} \cdot e^{\frac{m^{*} v^{2}}{2 k_{B} T_{o}}}
$$

The varying electric field $E(x)$ in the BTE emerging from the spatially-dependent doping density and carrier concentration, $N_{D}(\mathrm{x})$ and $n(x)$ is instantiated from the Poisson equation as,

$$
\frac{d^{2} \phi}{d x^{2}}=-\frac{d E}{d x}=-e \frac{N_{D}(x)-n(x)}{\in \epsilon_{0}}=-\rho(x)
$$

where $\in$ is the non-dynamic dielectric constant. The BTE and Poisson equations constitute a set of coupled, non-linear models, requiring eqs. (14-17) to be solved self-consistently since the electron density can be related to the distribution function as,

$$
n(x)=\int_{-\infty}^{+\infty} f(x, v) d v
$$

The simulation process is carried out self-consistently with the Poisson equation. Figure 5 shows the model of the carbon nanotube that gives a theoretically best control over the nanoscale channel. The model can be applied straightforwardly to a two-dimensional graphene provided that an additional gate (dual-gated) channel is created in order to form an ultra-thin graphene (see figure 5). The source and drain electrodes are heavily doped, and the gates modulates the conductance of the channel, akin to the conventional semiconductor materials.

The current-voltage characteristics of one-dimensional devices is strongly influenced by their quantum transport and electrostatics, so we implemented a self-consistent iteration between the BTE and Poisson equation. The general procedure is as follows; For a given carrier density, the Poisson equation is solved to obtain the electrostatic potential in the CNT channel. Next, the computed electrostatic potential is used as an input to the BTE, and an improved guess for the carrier density is obtained. The iteration process between the BTE and Poisson equation is repeated until self-consistency is achieved. Finally, the current for the self-consistent electrostatic potential is calculated.

For the model of figure 6, it is convenient to solve the Poisson equation in cylindrical coordinates. Since the electrostatic potential and the carrier density are invariant around the carbon nanotube, the Poisson equation is essentially a 2-D problem in the channel trajectory (x-direction) and radial to the channel (r-direction) as indicated in figure 6. Under this boundary, the Poisson equation can be expressed as,

$$
\Delta^{2} E_{m}(r, z)=-\frac{e}{\varepsilon} \rho
$$

where $\rho$ represents the carrier density, which could be relaxed to zero for grid points on the nanotube surface and non-zero otherwise, $E_{m}$ represents the energy level (state) minus the work function of the 
nanotube and is indeed the mid-point energy for the grid points on the nanotube surface. The electric field is relaxed to zero at $r=0$ for convenience [43]. Consequently,

$\left.\varepsilon_{r}\right|_{r=0}=0$

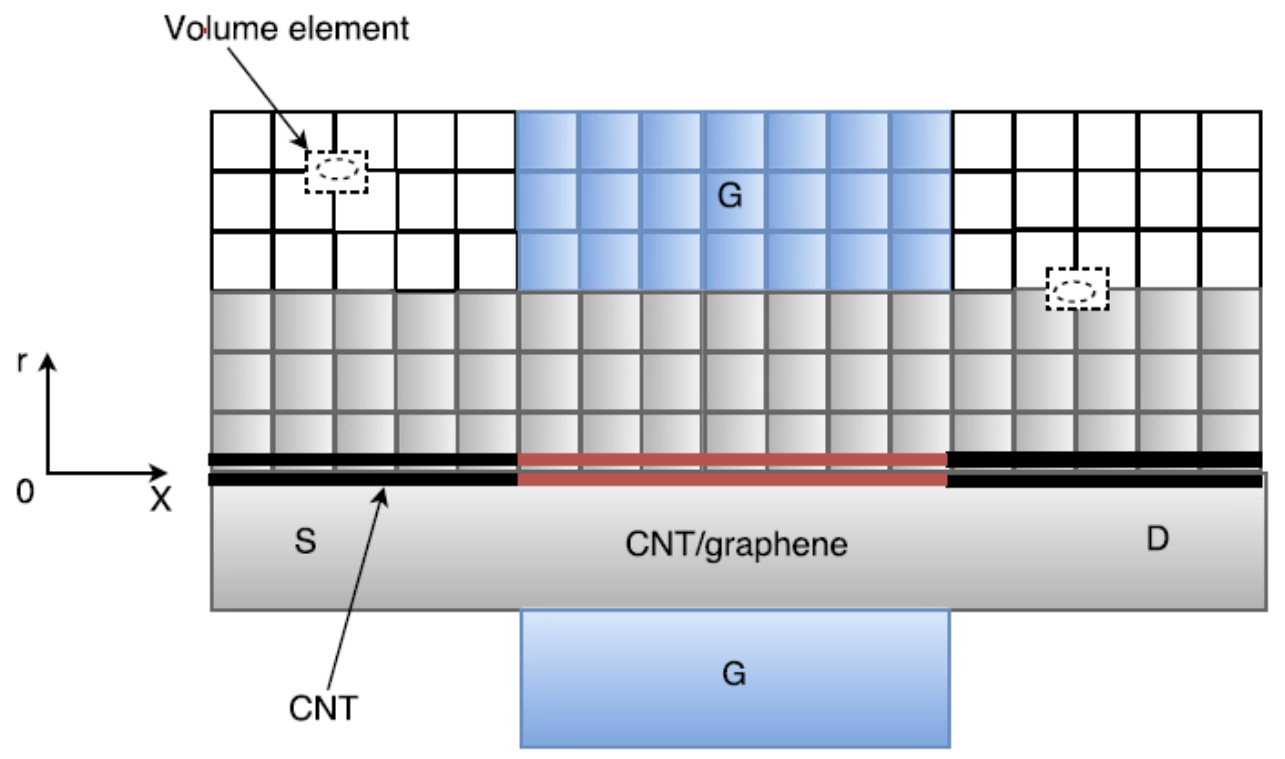

Figure 6: General model of the coaxially gated CNT with semi-infinite, heavily doped channel as the source/drain contacts. Also shown are the simulation grid, simulated area and a volume element assumed to be in cylindrical coordinate arrangement used for solving the Poisson equation. The dashed rectangular structure delineates the element adopted to linearize the Poisson equation at position (xi, rj).

Since we can predict the electrostatic potential at the gate channel, exploiting the Fermi level of the grounded channel as zero energy will lead to a gate channel electrostatic potential equivalent to,

$E_{m}($ gate $)=-e V_{G}+\phi_{m s}$

where $V_{G}$ is the applied bias, and $\phi_{m}$ is the difference in the work function between the gate electrode and the nanotube. By simulating a significantly massive area (see figure 6), the remaining boundaries can be subjected to the Neumann boundary conditions where the applied bias perpendicular to the boundary is relaxed to zero.

The continuous form of eq. (18) is then discretised for computer simulation. It is convenient to take a volume element near the grid point as shown in figure 6, and then subject it to the integral form of the Poisson equation, which is a ring around the nanotube axis with a rectangular cross section,

$$
\tilde{N} \cdot \vec{d} \vec{S}=q_{i j}
$$

where $q_{i j}$ is the charge in the volume element. The discretised equation for an element positioned at the point $\left(x_{i}, r_{j}\right)$ in space is given by,

$$
\begin{aligned}
& e r_{j} \Delta\left(N_{D}-n_{n e t}\right)=\varepsilon_{o}\left(\frac{r_{r-j}+}{2} \Delta x \frac{E_{m}^{i, j-1}-E_{m}^{i, j}}{\Delta r}+\frac{r_{j+1}+r_{j}}{2} \Delta x \frac{E_{m}^{i, j}}{2}\right)+ \\
& r_{j} \Delta r \frac{E_{m}^{i+l, j}-E_{m}^{i, j}}{\Delta x}+r_{j} \Delta r \frac{E_{m}^{i-l, j}-E_{m}^{i, j}}{\Delta x}
\end{aligned}
$$


Eq. (22) is linear and mathematically simple to solve, however, the convergence of the Poisson equation and carrier transport is poor. Therefore, a non-linear Poisson equation which associates the electrostatic potential through a non-linear dummy function to the charge carrier density is employed to improve the convergence of the BTE-Poisson loop. The non-linear dummy function correlating the carrier density to the electrostatic potential should be as comparable to the physical correlation provided by the carrier transport as possible for enhanced convergence. In most cases, a semi-classical, equilibrium carrier transport with a dummy quasi-Fermi level is employed as the dummy function. The non-linear Poisson portion is then solved for its electrostatic potential profile using Gummel iteration procedure. Details of the non-linear Poisson solver can be found in [44]. The coupling between the BTE and Poisson equation is stable if the differential between the old and new potential profiles decreases monotonically with successive iteration loops for all applied electric fields.

The convergence is smooth and stable down to a miniscule approximation error of $10^{-5} \mathrm{~V}$, which is analogous to the convergence of self-consistent drift-diffusion simulations. This is in conspicuous contrast to Monte Carlo simulation approach which exhibits noise in its convergence and thus makes it difficult to decrease the error rate below $k_{B} T_{L} / e$ threshold. It should be noted that every time the iteration loop returns back to solve the BTE, the default solution of the BTE is employed as starting guess. This shortens the number of iterations required to solve the BTE in successive loops. Hence, there is substantial savings in the time required for self-consistent iterations because the BTE is the most time-consuming path of the loop. Next, we discuss the direct implementation of this procedure on a $50 \mathrm{~nm}$ single-wall carbon nanotube.

It has been reported that present-day microelectronic devices operate at approximately 50 percent of the ballistic threshold [45], and that after scaling to nanometer scale range, they operate at over 80 percent of the ballistic limit [46]. This is not surprising given that carriers in these nanosystems have finite mobilities and mean free paths. The reason for this is that there exists a critical region of the channel near the source, where carrier motion becomes nearly ballistic. This is called quasi-ballistic transport [47-49]. Although this effect has been studied before, with approximate solutions to the BTE [50], this work is the first attempt to investigate the quasi-ballistic transport in one-dimensional carbon nanotubes by solving the BTE without passing through the approximation stage. The solutions from the BTE are then compared with the solutions from the ballistic BTE as well as solutions from standard macroscopic models.

The standard macroscopic models used in this study are the electron transport (ET), hydrodynamic (HD) and drift diffusion (DD). These models are adjusted to low-field velocity, symmetric velocity versus field curve, and in the case of ET and HD, energy versus field curve of bulk carbon nanotube. This adjustment guarantees that the transport models represent homogeneous physical problems but takes varying implementation assumptions. The electron transport and hydrodynamic models are solved using the non-oscillatory method and the drift diffusion model is solved using the well-known Scharfetter-Gummel formalism [51].

\section{RESULTS AND DISCUSSION}

The current-voltage characteristics for the BTE, ballistic BTE, drift diffusion, hydrodynamics and electron transport models is shown in figure 7. Surprisingly, the current from the DD is found to be very close to the current from the BTE, even though the DD is not an ideal representation of carrier transport at these length scales. The ET and HD models exhibit increased currents with the ET model even exhibiting more current than the current from the ballistic BTE. This can be attributed to the ensemble average velocities inside the carbon nanosystems. 


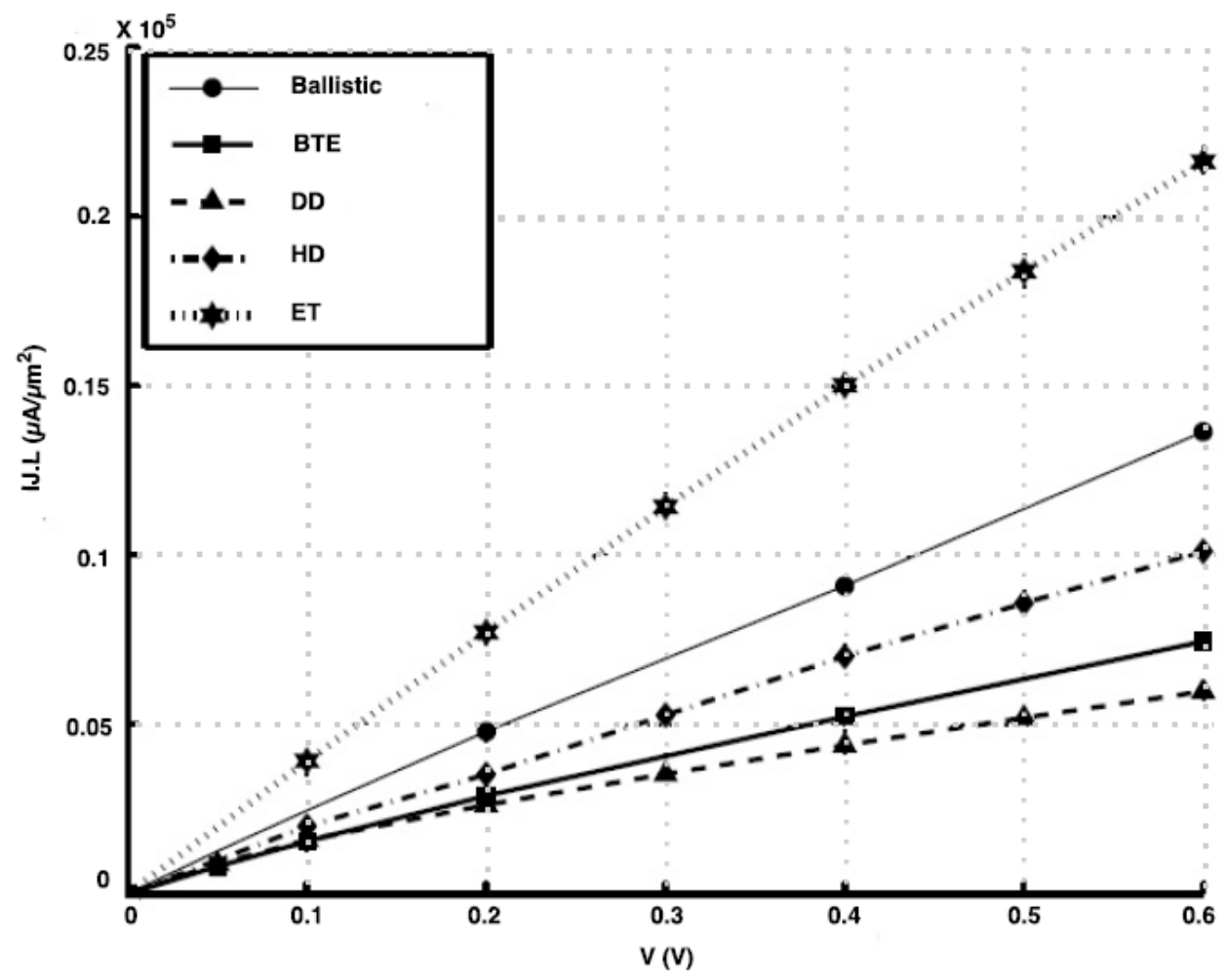

Figure 7: The current-voltage (I-V) characteristics of the ballistic, BTE, DD, HD and ET models.

The ensemble velocity average from the BTE solution for increasing applied electric field is shown in figure 8. It is conspicuous that, although there is significant velocity overshoot in the system, the velocity at the source-drain interface approximates the thermal velocity of the sample, $1 \times 10^{7} \mathrm{~cm} / \mathrm{s}$. This is a signature feature of quasi-ballistic transport.

The comparison of the average ensemble velocity for the represented transport models for $0.6 \mathrm{~V}$ applied bias is shown in figure 9. The velocity from the DD model is found to be highly unphysical because it clipped to the saturation velocity inside the nanodevice. At the same time, the thermal velocity from the BTE turns out to be numerically comparable to the saturation velocity from the DD model. Accordingly, the quantum of currents from the BTE and DD turns out be nearly the same. In addition, the velocity from the BTE is found to be smaller than the velocities from the ET and HD. The HD model was found to exhibit spurious velocity overshoot near the drain channel which is unphysical but a mathematical relic of the HD model. On the contrary, although the ET model does not exhibit any spurious velocity overshoot, it does promote higher velocity near the source-drain interface than the thermal velocity, which is also unphysical. However, the ballistic BTE is found to be limited by the thermal velocity, although it exhibits unreasonably high velocity.

From the forgoing results, it is conspicuous that ET, HD and DD failed to regenerate the characteristics of one-dimensional nanotubes. While the argument against the DD model is unambiguous, the argument against the ET and HD is ambiguous because the implementation variables in ET and HD models are typically unphysical constants, and as such can be modulated to match the features of a wide variety of devices. However, our goal is not to propose a preferable tuning of variables but to demonstrate that macroscopic models cannot not be used to describe quasi-ballistic transport in one-dimensional single-wall carbon nanotubes. 


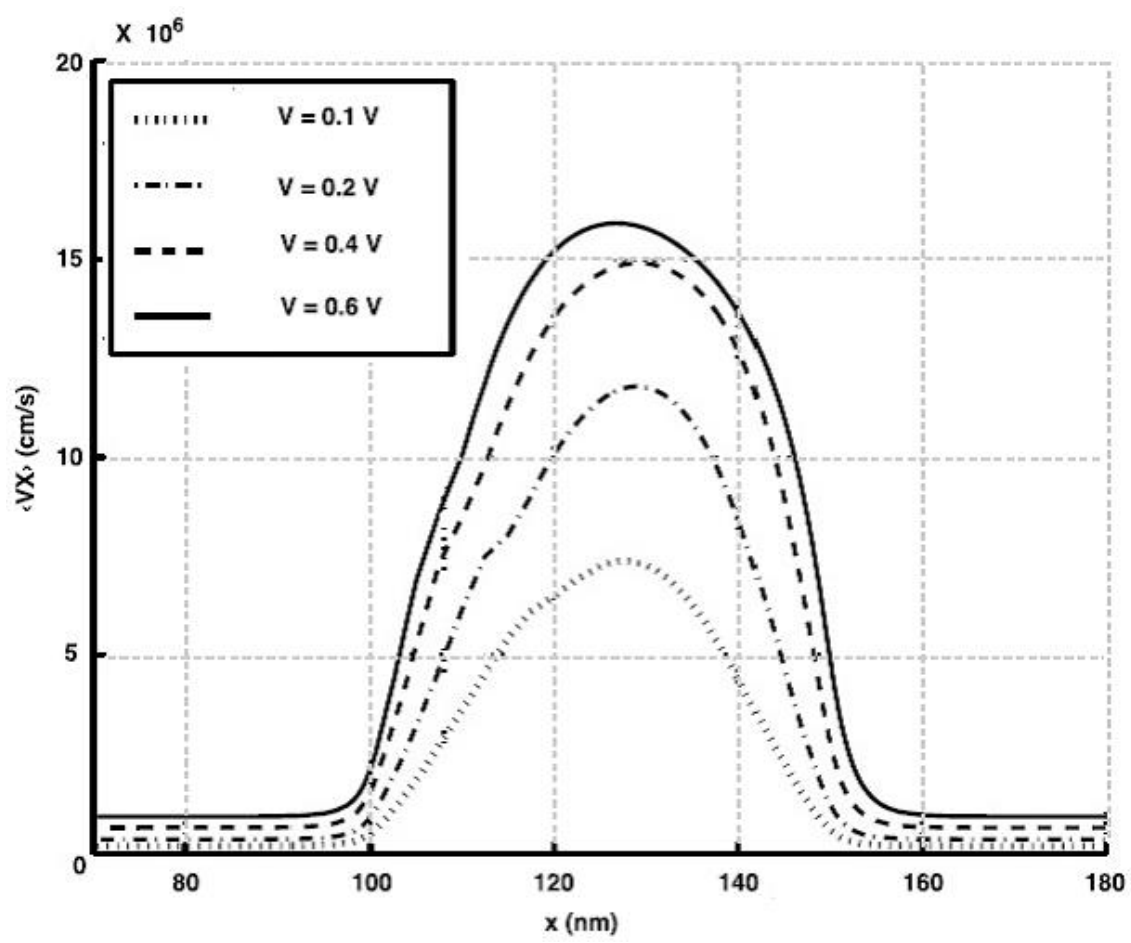

Figure 8: Ensemble velocity average from BTE solution at high bias

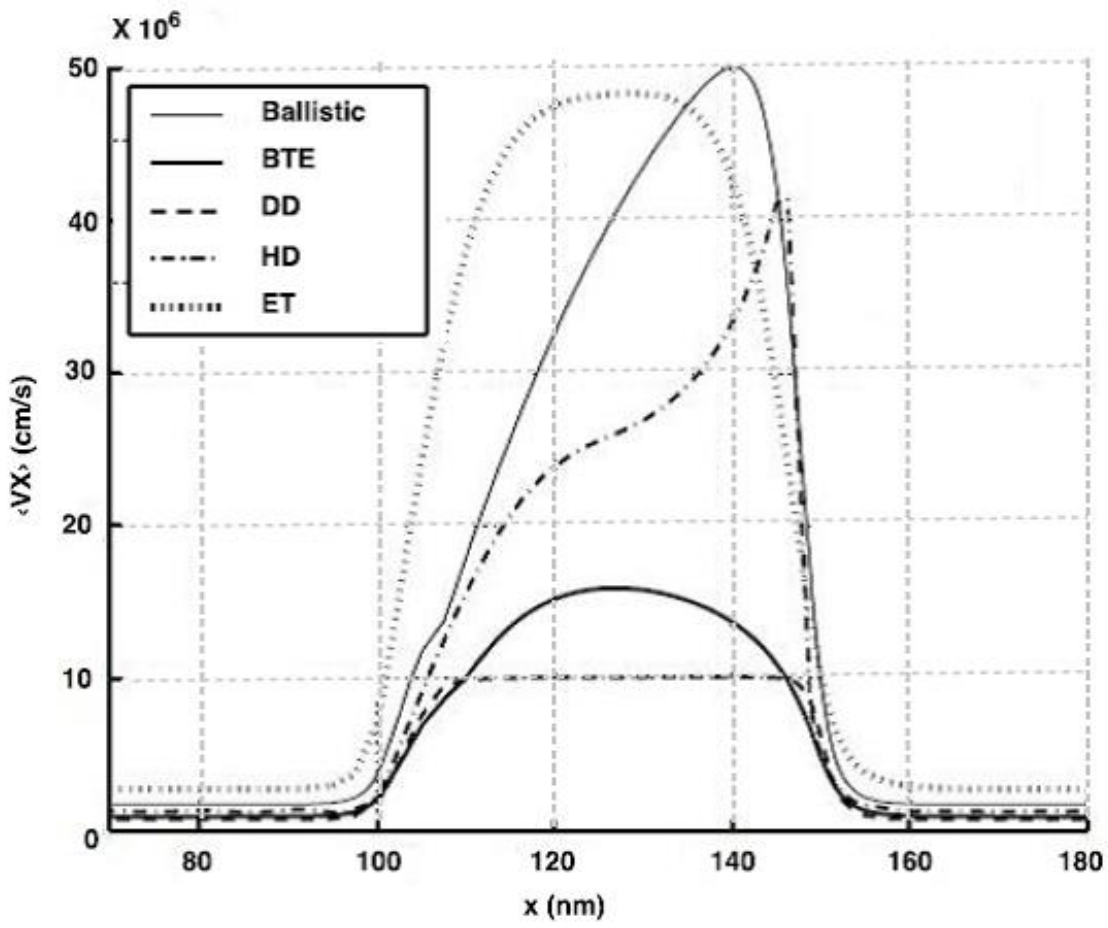

Figure 9: Ensemble velocity average for the ballistic, BTE, DD, HD and ET solutions at high bias 
The current-voltage characteristics of the carbon nanotube benchmarked on an experimental investigation is shown in figure 10. Also shown is the plot of the experimental data [26]. The different temperatures were extracted from degenerate statistics [52] as nondegeneracy is impractical in this regime. The simulation is implemented from $-5 v$ to $+5 v$. Ordinarily, the negative applied bias does not influence the outcome as symmetry is assumed. Surprisingly, the current-voltage characteristics was found to overlap at the simulated temperatures, suggesting that the current-voltage characteristics is independent of temperature. From the result, it is obvious that three regimes of nanoscale operation are possible - ohmic region, non-ohmic region and saturation region. The current-voltage characteristics is found to be linear in the low-bias regime, consistent with ohm's law for microelectronic devices. However, as the applied voltage increases, the current-voltage characteristics declines suggesting a trend of decreasing conductance. This is the nonohmic regime because ohm's law cannot apply in this regime. Further increase in the applied electric field eventually causes the current-voltage characteristics to saturate to its saturation current $I_{\text {sat }}$. This is called the saturation region.

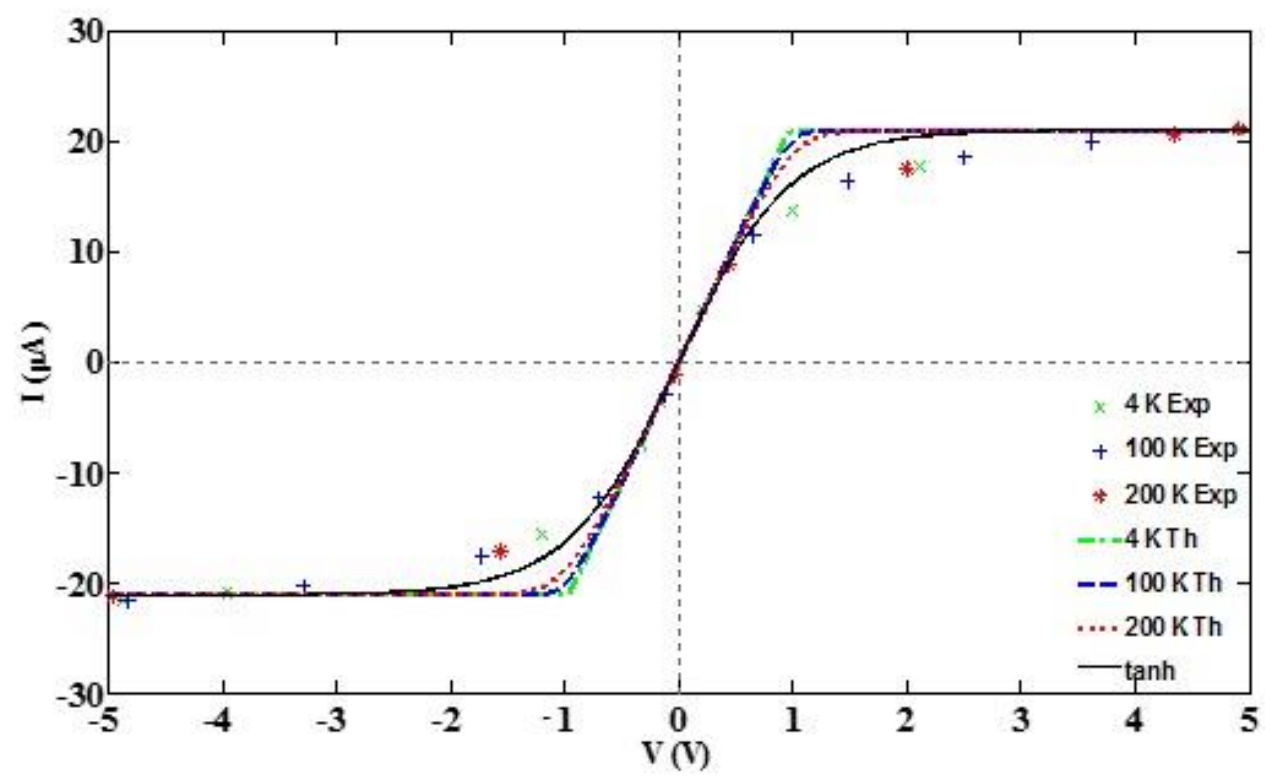

Figure 10: Current-voltage characteristics of a single-wall carbon nanotube. Also simulated is the experimental Data [26].

The saturation of current with increasing electric field can be explained from the perspective of electronic bandstructure of carbon nanotubes. It is known that the current in metallic one-dimensional carbon nanotubes is fetched by the two propagating 1-D subbands. Under ballistic conditions, the applied electric field of the left and right moving states differs by $e V$. At moderate applied bias, this results in ohmic response. However, when the applied bias exceeds the Fermi energy of the 1-D subbands, the left moving states will be completely depleted, and the current will saturate to its saturation value $I_{\text {sat }}$.

As the current-voltage characteristics no longer keeps faith with ohm's law in the sublinear regime, defining the actual resistance of the carbon nanotube becomes challenging. This requires us to distinguish between direct resistance, $R=V / I$ (due to ohm's law) and indirect resistance otherwise called differential or incremental resistance, $r=d V / \mathrm{dI}$. Applying David et al. [53] principle, the current $I$ flowing through a carbon nanotube is given by,

$$
I=I_{s a t} \tanh \left(V / V_{c n}\right)=\left(V_{c n} / R_{o}\right) \tanh \left(V / V_{c n}\right)=\left(V / R_{o}\right) \tanh \left(V / V_{c n}\right) /\left(V / V_{c n}\right)
$$


where $I_{s a t}$ is the saturation current and tanh is the hyperbolic tan function. The indirect resistance is given by the differential coefficient of the applied bias $V_{n(p)}$ with respect to the applied current $I_{n(p)}$, thus $r_{n(p)}=d V / \mathrm{dI}_{n(p)}$. The actual resistance associated with the signal propagation through the nanotube is determined by the dc bias. Thus, the direct $R$ and incremental $r$ resistances in the sublinear region can be expressed as,

$$
\begin{aligned}
& R_{n(p)}=V / I_{n(p)}=R_{o n(p)}\left[1+\left(V / V_{c}\right)^{\gamma_{n(p)}}\right]=R_{o n(p)} /\left[1-\left(I_{n(p)} / I_{\text {satn }(p)}\right)^{\gamma_{n(p)}}\right]^{1 / \gamma_{n(p)}} \\
& r_{n(p)}=d V / \mathrm{d} I_{n(p)}=R_{o n(p)}\left[1+\left(V / V_{c}\right)^{\gamma_{n(p)}}\right]^{1+\left(1 / \gamma_{n(p)}\right)}
\end{aligned}
$$

The direct and differential resistances as a function of the applied electric field, and the direct and differential resistances as a function of the length of the nanotube for $V=V_{c}$ are shown in figures 11 and 12 respectively. Surprisingly, the resistance is found to be limited to its ohmic value when the ratio of the applied bias to the critical voltage is less than unity $\left(V / V_{c}<1\right)$, but rises dramatically when the applied voltage towers over its critical value, such that $V / V_{c}>1$. In addition, the differential resistance is found to rise faster than the direct resistance.

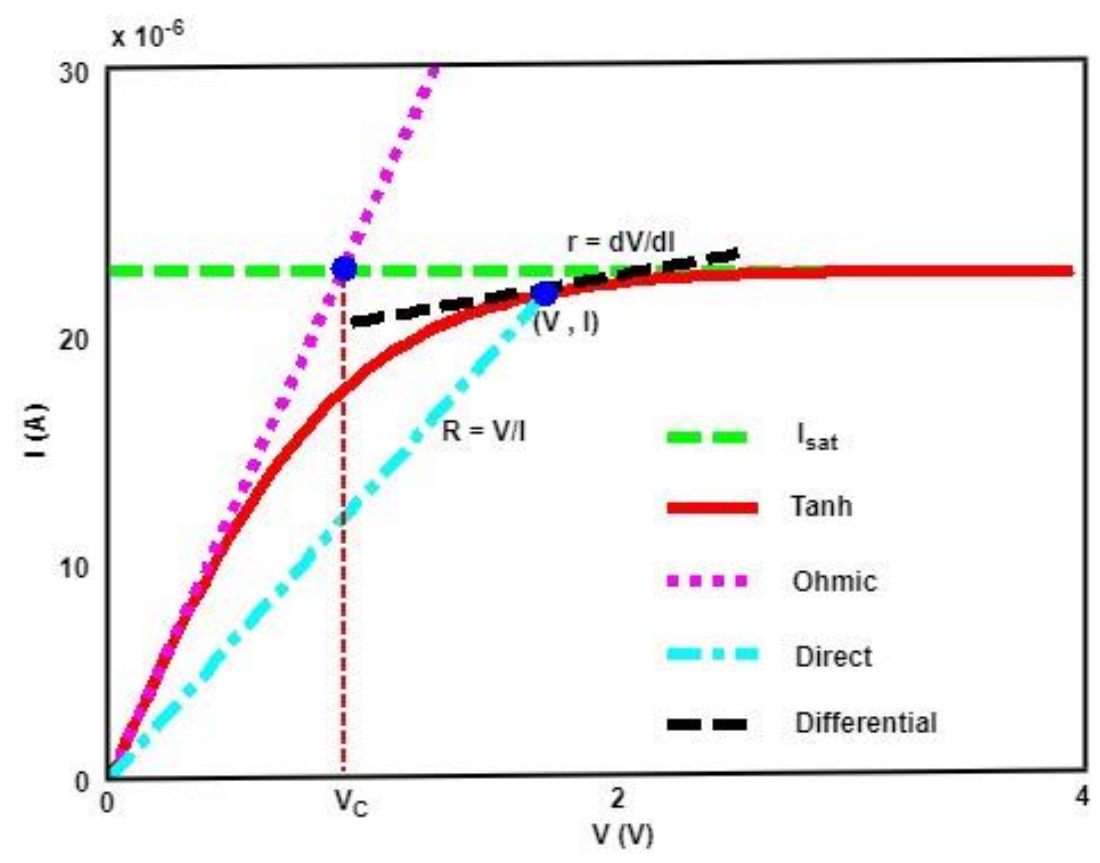

Figure 11: Direct and incremental resistance as a function of applied voltage. Also shown is the hyberbilc tanh.

These findings have practical implications in device design and performance. To distil new insights from these implications, we need to further probe the fundamental laws of electrical circuit design. Ohm's law is at the heart of circuit theory both for analogue and digital electronic circuit designs. Basically, Ohm's law describes the linear current response to the applied bias across the length of a conductor. The inverse gradient of the current-voltage characteristics or resistance is a constant that is extensively discussed in the literature. However, the linear response to the electric field changes to a non-linear response with current eventually saturating to a finite value $I_{\text {sat }}$, which leads to the 
breakdown of ohm's law. The aforementioned breakdown in ohm's law substantially affects the flow of transport carriers in the carbon nanotube. Furthermore, the surge in direct and incremental resistance changes the current and voltage division laws, time constants and power consumption, all of which play essential roles in device design and performance. Additionally, the transient resistance-capacitance $(R C)$ switching delay is also severely affected by the surge in resistance due to the aforementioned breakdown.

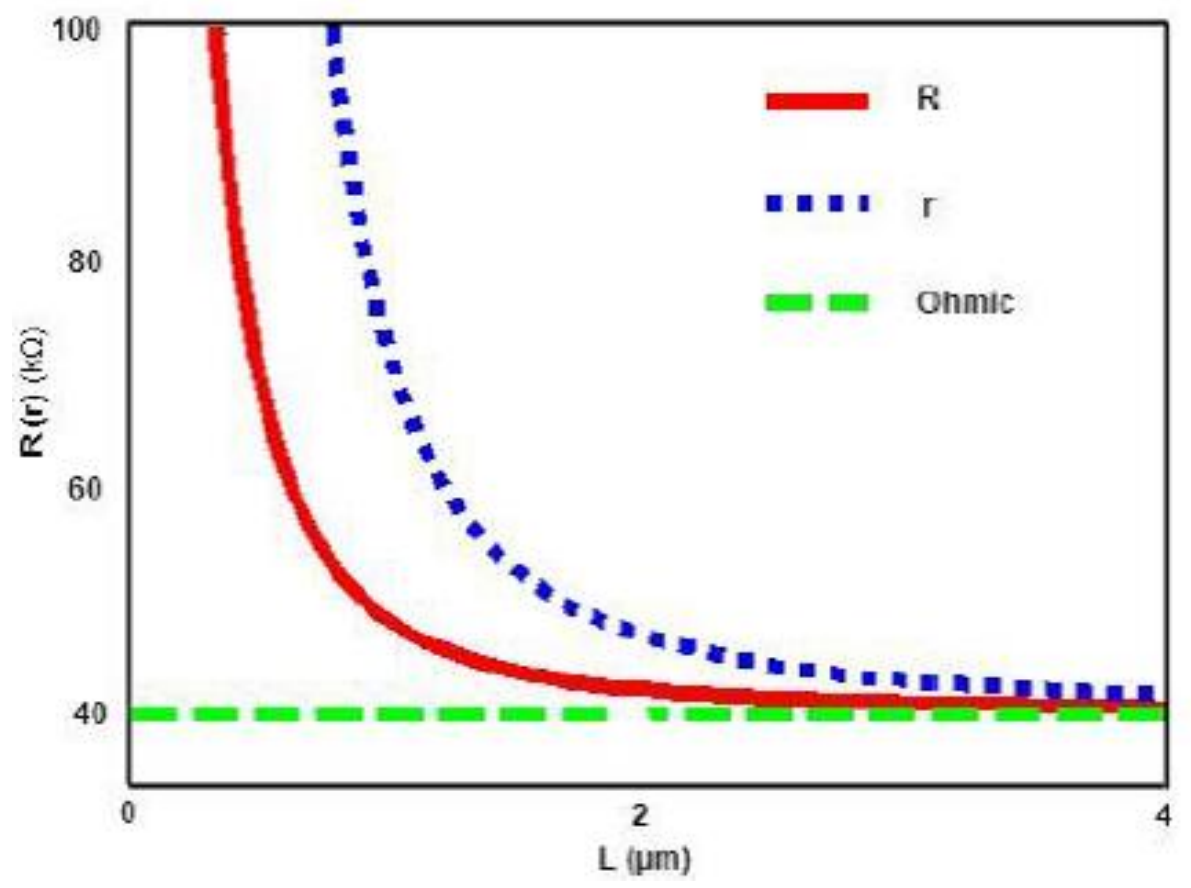

Figure 12: Direct and incremental resistance as a function of channel lengths for $\mathrm{V}=\mathrm{Vc}$

\section{CONCLUSION}

Following the first demonstration of carbon-based devices, the understanding of 1-D carbon nanotubes (CNTs) is evolving, and their performance in device applications is improving rather rapidly. Leading this improvement is the understanding of carrier transport in these devise when they are under the influence of varying electric fields.

This work has demonstrated the first ever direct solution of the Boltzmann Transport Equation (BTE) for single-wall carbon nanotubes that can be employed for practical device simulations. This was achieved by leveraging powerful theoretical models to discretize the BTE both in energy and momentum without passing through the approximation phase. This approach is not only fast but also has low computational and memory requirements. The approach reported in this work was found to be appropriate for self-consistent device simulations because it exhibited smooth and stable convergence when coupled to Poisson model. Finally, this approach was implemented on a 50nm single-wall carbon nanotube. This implementation demonstrated that one-dimensional nanoscale single-wall carbon nanotubes operate in ballistic and quasi-ballistic regimes where carrier transport is ballistic or near ballistic across a nanoscale region of the device near the source channel. Standard macroscopic models were found not to hold in this regime because their transport is predicated on classical assumptions. 


\section{REFERENCES}

[1] Bejenari I., Claus M. "Analytical Model of One-Dimensional Ballistic Shottky-Barrier Transistors", IEEE Transactions on Electron Devices, arXiv:1703.05092v2 [cond-mat.meshall], 2017.

[2] Lopez-Bezanilla A. "Electronic Transport Properties of Chemically Modified Double-Walled Carbon Nanotubes", arXiv:1603.08030v1 [cond-mat.mtrl-sci], 1-7, 2016.

[3] $\mathrm{Xu} \mathrm{F.,} \mathrm{Yu} \mathrm{Z.,} \mathrm{Gong} \mathrm{Z.,} \mathrm{Jin} \mathrm{H.} \mathrm{"First-principles} \mathrm{study} \mathrm{on} \mathrm{the} \mathrm{electronic} \mathrm{and} \mathrm{transport}$ properties of periodically nitrogen-doped graphene and carbon nanotube superlattices", Frontiers of Physics 12, 127306, 2017.

[4] Teichert F., Zienert A., Schuster J., Schreiber M. "Electronic transport inmetallic carbon nanotubes with mixed defects within the strong localization regime', Computational Materials Science 138, 49-57, 2017.

[5] Mirza M. M., Schupp F. J., Mol J. A., MacLaren D. A., Briggs G. A. D., Paul D. J. "One dimensional transport in silicon nanowire junction-less field effect transistors", Scientific Reports 7, 3004, 2017.

[6] Milovanovic S. "Electronic transport properties in nano- and micro-engineered graphene structures", PhD dissertation, Universiteit Antwerpen, Belgium, 2017.

[7] Kishimoto K., Okada S. "Electronic structure of bilayer graphene with defects under an external electric field", Japanese Journal of Applied Physics 56, 06GE01, 2017.

[8] Kaiser B., Park Y. W., Kim G. T., Choi E. S., Dusberg G., Roth, S. “"Electronic transport in carbon nanotube ropes and mats", Synthetic Metals 103, 2547-2550, 1999.

[9] Salvato M., Cirillo M., Lucci M., Orlandducci S., Ottavian I., Terranova M. L., Toschi F. "Charge-transport and tunnelling in single-walled carbon nanotube bundles", In Physical Review Letters 101, 246804, 2009.

[10] Zhang J., Terrones M., Park C. R, Mukherjee R., Monthioux M., Koratkar N., Kim Y. S., Hurt R., Frackowiak E., Enoki T., Chen Y., Chen Y., Bianco A. "'Carbon science in 2016: Status, challenges and perspectives", Carbon 98, 708-732, 2016.

[11] Villanueva J .R., Olivares M. "Gravitational Rutherford scattering and Keplerian orbits for electrically charged bodies in heterotic string theory', Eur. Phys. J. C 75, 562, 1-12, 2015.

[12] Khalil O. "Theoretical Atomic Model and the Theory of Everything', International Journal of Physics 5 87-91, 2017.

[13] Ozdemir M. D., Atasever O., Ozdemir B., Yarar Z., Ozdemir M. "A comparative study of transport properties of monolayer graphene and AIGaN-GaN heterostructure", AIP Advances, 5, 077101, 2015.

[14] Roch A., Stepien L., Roch T., Dani I., Leyens C., Jost O., Leson A. “Optical absorption spectroscopy and properties of single walled carbon nanotubes at high temperature', Synthetic Metals 197, 182-187, 2014.

[15] Chandra J., Bhatt P. K., Kholiya K. "Analysis of Equation of State for Carbon Nanotubes", Journal of Nanotechnology 2013(639068), 1-5, 2013.

[16] Praneuski A., Tivanov M., Czarnacka K., Kierczynski K. "Two-dimensional thermal conductivity of defect-free Single-wall carbon nanotubes', In Przeglad Elektrotechniczny, 8184, 2016.

[17] Mceuen P. L., Park J.-Y. "Electron Transport in Single-Walled Carbon nanotubes", MRS Bulletin 29, 272, 2004.

[18] Davoody A. H., Karimi F., Arnold M. S., Knezevic I. "Theory of Exciton Energy Transfer in Carbon Nanotube Composites", arXiv:1604.04648v2 [cond-mat.mes-hall], 2016.

[19] Tang Y., Lu J., Liu D., Yan X., Yao C., Zhu H. "Structural Derivative and Electronic Property of Armchair Carbon Nanotubes from Carbon Clusters", Journal of Nanomaterials 2017, 1-11, 2017.

[20] Xu Z. "Heat transport in low-dimensional materials: A review and perspective", Theoretical and Applied Mechanics Letters, 6, 113-121, 2016. 
[21] Datta S. "Electronic Transport in Mesoscopic Systems". Cambridge University Press, Cambridge, London, 1995.

[22] Li K., Eres G., Howe J., Chuang Y.-J., Li X., Gu Z., Zhang L., Xie S., Pan Z. "SelfAssembly of Graphene on Carbon Nanotube Surfaces", Scientific Reports 3, 2353, 1-4, 2013.

[23] Shi Z., Hong X., Bechtel H. A. Zeng B., Martin M. C., Watanabe K., Taniguchi T., Shen Y.R., Wang F. "Observation of a Luttinger-liquid plasmon in metal single-walled carbon nanotubes", Nature Photonics, 9, 515-519, 2015.

[24] Maslov D. L., Sone M. "Conductance of Luttinger-Liquid Wires Connected to Reservoirs". arXiV:cond-mat/9603106v1, 1-11, 1996.

[25] Roche S., Jiang J., Foa Torres L. E. F., Saito R. "'Charge transport in carbon nanotubes: quantum effects of electron-phonon coupling”, J. Phys. Condens. Matter 19, 1-22, 2007.

[26] Yao Z. Kane C. L., Dekker C. "High-field Electrical Transport in Single-wall Carbon Nanotubes", Physical Review Letters, 84, 2941-2944, 2000.

[27] Vos M., Grande P. L. "The relation between the electron energy loss spectra of hafnia and its dielectric function', Surface Science 630, 1-8, 2014.

[28] Javey A., Guo J., Paulsson M., Wang Q., Mann D. Lundstrom M., Dai H. "High-field quasi-ballistic transport in carbon nanotubes", Phys. Rev. Lett., 92, 1-17, 2004.

[29] Jovanovi V. B., Borka D., Galijas S. M. D. "Channeling of protons through radial deformed carbon nanotubes", Physics Letters A 381, 1-6, 2017.

[30] Gautreau P., Ragab T., Basaran C. "Hot phonons contribution to Joule heating in single- wall carbon nanotubes", J. Appl. Phys. 112, 1-8, 2012.

[31] Pop E., Mann D., Wang Q., Goodson K., Dai H. "Thermal Conductance of an Individual Single-Wall Carbon Nanotube above Room Temperature', Nano Letters 6, 96-100, 2006.

[32] Li J., Miranda H. P. C., Niquet Y.-M., Genovese L., Duchemin I., Wirtz L., Delerue C. "Phonon-limited carrier mobility and resistivity from carbon nanotubes to graphene", In Physical Review B, 1-13, 2015.

[33] SchieBI S. P., de Vries X., Rother M., Masse A., Brohmann M., Bobbert P. A., Zaumseil J. "Modelling carrier density dependent charge transport in semiconducting carbon nanotube networks", Physical Review Materials 1, 1-13, 2017.

[34] Kumar G., Singh M., Bulusu A., Trivedi G. "'A framework to simulate semiconductor devices using parallel computer architecture”, J. Phys. : conf. Ser., 759, 012098, 2016.

[35] Sharma D., JAGGI N. "Vibrational spectra and phonon dispersion analysis of a single walled zigzag carbon nanotube: A first principles study", In Canadian Journal of Physics 94, 1112 $1118,2016$.

[36] Abdulllah H. Y. "Theoretical study of the binding energy of some gases on Al-doped carbon nanotube', Results in Physics, 6, 1146-1151, 2016.

[37] Evarestov R. A., Bandura A. V., Porsev V. V. "Thermodynamic properties of nanotubes: zonefolding approach", Lithuanian Journal of Physics, 56, 164-172, 2016.

[38] Davoody A. H., Karimi F., Arnold M. S., Knezevic I. "'Unexpectedly Fast Phonon-Assisted Exciton hopping between Carbon Nanotubes”, J. Phys. Chem. C 121, 13084-13091, 2017.

[39] Park S., Woo S., Mele E. J., Min H. 'Semiclassical Boltzmann transport theory for multi-Weyl semimetals", arXiv:1701.07578v2 [cond-mat.mes-hall], 1-16, 2017.

[40] Dasgupta A., Chauhan Y. S. "Modeling of Flicker Noise in Quasi-ballistic FETs", In Simulation of Semiconductor Processes and Devices, 105-108, 2017.

[41] Zhang F., Hou P.-X., Liu C., Wang B.-W., Jiang H., Chen M.-L., Sun D.-M., Li J.-C., Cong H.-T., Kauppinen E. I., Cheng H.-M. "'Growth of semiconducting single-wall carbon nanotubes with a narrow bandgap distribution", Nature Communications, 7, 1-9, 2016.

[42] Zhou Y., Fan Z., Qin G., Yang J.-Y., Ouyang T., Hu M. "Methodology perspective of computing thermal transport in low-dimensional materials and nanostructures: The old and new', ACS Omega 3, 3278-3284, 2018. 
[43] Auth C. P., Plummer J. D. "Scaling theory for cylindrical, fully-depleted surrounding-gate MOSEFET's', IEEE Electron Device Letters, 18, 74-76, 1997.

[44] Qiao Z.-H., Li Z.-L., Tang T. "A finite difference scheme for solving the non-linear PoissonBoltzmann equation modelling charged spheres", Journal of Computational Mathematics, 24, 252-264, 2006.

[45] Assad F., Ren Z., Datta S., Lundstrom M. S., Bendix P. M. "Performance limits of silicon MOSFETs", In IEDM Technical Digest. IEEE Electron Devices Society, Piscataway NJ. Cat.99CH36318, 547-550, 1999.

[46] Timp G., Bude J., Bourdelle K. K., Garno J., Ghetti A., Gossmann H., Green M., Forsyth G., Kim Y., Kleiman R., Klemens F., Kornblit A., Lochstampfor C., Mansfield W., Moccio S., Sorsch T., Tennant D. M., Timp W., Tung R. "The Ballistic Nano-transistor', In IEDM Technical Digest, Cat. 99CH36318, 55-58, 1999.

[47] Dasgupta A., Agarwal A., Chuhan Y. S. “'An Improved Model for Quasi-Ballistic Transport in MOSFETs", IEEE Transactions on Electron Devices 64, 3032-3036, 2017.

[48] Upadhyay A. K., Kushwaha A. K., Vishvakarma S. K. “A Unified Scalable Quasi-Ballistic Transport Model of GFET for Circuit Simulations", IEEE Transactions on Electron Devices 65, 739-746, 2018.

[49] Tanaka H., Suda J., Kimoto T. "Impacts of energy relaxation process on quasi-ballistic hole transport capability in germanium and silicon nanowires", Journal of Applied Physics 123, $024305,1-8,2018$.

[50] Anile M. A., Carrillo J. A., Gamba I. M., Shu C.-W. "Approximation of the BTE by a Relaxation-time Operator: Simulations for a $50 \mathrm{~nm}$-channel Si Diode', VLSI Design, 13, 349354, 2001.

[51] Koprucki T., Kantner M., Fuhrmann J., Garner K. "On modifications of the ScharfetterGummel scheme for drift-diffusion equations with Fermi-like statistical distribution functions", In Numerical Simulation of Optoelectronic Devices (NUSOD), 14705086, 155-156, 2014.

[52] Makarovski A., An L., Liu J., Finkelstein G. "Persistent orbital degeneracy in carbon nanotubes"'. Physical review B, 74, 1-6, 2006.

[53] Chen X. "Modelling of Experimental Adsorption Isotherm Data", Information 6, 14-22, 2015. 